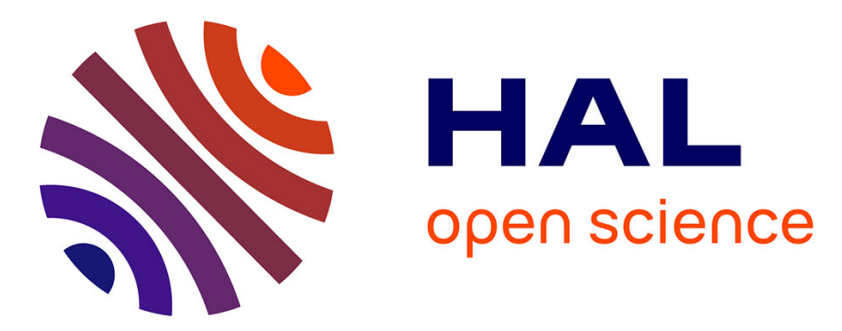

\title{
Electron-impact single and double ionization of W17 +
}

J Rausch, A Becker, K Spruck, J Hellhund, A Borovik, K Huber, S Schippers, A Müller

\section{To cite this version:}

J Rausch, A Becker, K Spruck, J Hellhund, A Borovik, et al.. Electron-impact single and double ionization of W17 +. Journal of Physics B: Atomic, Molecular and Optical Physics, 2011, 44 (16), pp.165202. 10.1088/0953-4075/44/16/165202 . hal-00645375

\section{HAL Id: hal-00645375 https://hal.science/hal-00645375}

Submitted on 28 Nov 2011

HAL is a multi-disciplinary open access archive for the deposit and dissemination of scientific research documents, whether they are published or not. The documents may come from teaching and research institutions in France or abroad, or from public or private research centers.
L'archive ouverte pluridisciplinaire $\mathbf{H A L}$, est destinée au dépôt et à la diffusion de documents scientifiques de niveau recherche, publiés ou non, émanant des établissements d'enseignement et de recherche français ou étrangers, des laboratoires publics ou privés. 


\title{
Electron-impact single and double ionization of $\mathrm{W}^{17+}$
}

\author{
J. Rausch, A. Becker, K. Spruck, J. Hellhund, A. Borovik Jr., \\ K. Huber, S. Schippers, and A. Müller $\ddagger$ \\ Institut für Atom- und Molekülphysik, Justus-Liebig-Universität Giessen, D-35392 \\ Giessen, Germany
}

\begin{abstract}
.
A crossed-beams setup was used to measure cross sections for electron-impact single and double ionization of $\mathrm{W}^{17+}$ ions. Absolute data and high-resolution scan spectra were obtained at collision energies ranging from threshold up to $1000 \mathrm{eV}$. Comparison of the experimental results with theoretical calculations for direct ionization suggests substantial contributions of excitation-autoionization processes to electron-impact single ionization of $\mathrm{W}^{17+}$.
\end{abstract}

PACS numbers: $34.80 . \mathrm{Dp}, 52.20 . \mathrm{Fs}$

Draft for J. Phys. B: At. Mol. \& Opt. Phys: 15 June 2011

$\ddagger$ Corresponding author, E-mail: Alfred.Mueller@iamp.physik.uni-giessen.de 


\section{Introduction}

Tungsten with its low sputtering yield, low chemical erosion, low tritium retention, high durability against neutron flux and good thermo-mechanical properties is presently considered an indispensable material for plasma facing components of future fusion reactors [1]. With tungsten exposed to the plasma edge $\mathrm{W}^{q+}$ impurities in the plasma are unavoidable [2]. Since high-Z materials do not get fully ionized in the fusion plasma strong emission of X-ray and EUV line radiation is expected [3, 4] in addition to bremsstrahlung from energetic-electron - highly-charged-ion collisions. The comparatively low-density plasma cannot reabsorb such radiation so that a great deal of energy badly needed for starting controlled self-sustained thermonuclear burning is lost from the plasma. Concentrations of less than $0.02 \%$ of tungsten ions already inhibit ignition of a deuterium-tritium plasma [2].

Understanding the influence of $\mathrm{W}$ as a plasma impurity and its impact on the plasma requires detailed knowledge about atomic processes and atomic structures of tungsten atoms in all stages of ionization [4]. Unfortunately, experimental atomic data for high-Z elements are scarce and theoretical calculations are difficult because of the pronounced many-electron and relativistic effects in heavy ions. Thus, the existing theoretical results are very uncertain for these ions. Obviously, experimental data are needed for testing and guiding new theoretical developments required for improving the predictive power of calculations for many-electron atoms and, consequently, of plasma modeling. This is especially true for tungsten where theoretical treatments are complicated by the presence of open d or f shells.

The atomic processes of prime interest in fusion research are electron-impact ionization and excitation as well as electron-ion recombination [5]. In comparison, photoionization is of reduced importance in fusion plasmas. However, the investigation of photoionization of tungsten ions provides useful information towards the role of tungsten atoms and ions in a plasma and can deliver valuable insights into their spectroscopic properties. Motivated by the renewed interest of the fusion community in tungsten, a comprehensive attempt is being made by our group to carry out measurements on electron-ion recombination [6], on electron-impact ionization and on photoionization [7] of tungsten ions in low to intermediate charge states. Single and multiple ionization of $\mathrm{W}^{q+}$ ions $(q=1, \ldots, 10)$ by electron impact have been investigated previously $[8,9,10]$. Theoretical calculations of plasma rate coefficients for electron-impact single ionization of $\mathrm{W}^{q+}$ ions were performed for all charge states $q$ by Loch et al [11] employing the configuration-average distorted-wave (CADW) approach. Unfortunately, only few cross section results are provided in the publication [11] and, in particular, no data are available for comparison with the present work.

The present paper reports experimental data for electron-impact single and double

ionization of the $\mathrm{W}^{17+}$ ion at collision energies up to one $\mathrm{keV}$. Absolute cross sections and high-resolution energy-scan spectra were obtained. 


\section{Experiment}

\subsection{General description of the setup and measurement procedures}

The present measurements were performed employing an electron-ion crossed-beams setup which has been used for electron-impact ionization experiments with various atomic ions $[12,13,14,15,16,17,18]$. Some details of the setup and the measurement procedures have been provided previously by Jacobi et al and Borovik et al [13, 17]. Here we provide details relevant to the particular ion under study. Emphasis is put on the discussion of cross section determination and the resulting error budget.

The $\mathrm{W}^{17+}$ target ions were produced by a $10 \mathrm{GHz}$ all-permanent-magnet electroncyclotron-resonance (ECR) ion source [19] operating at a microwave power of up to $100 \mathrm{~W}$. Tungsten hexacarbonyl, $\mathrm{W}(\mathrm{CO})_{6}$, was used as parent material. A small container separated from the ion source by a fine-regulation valve was filled with $\mathrm{W}(\mathrm{CO})_{6}$ powder which has a vapor pressure of about 0.02 mbar at room temperature [20]. Air was removed from the container by briefly opening an additional valve to a forepump. Gas flow into the ion source was regulated by the remotely controlled fine-regulation valve.

Ions were extracted from the ion source using an acceleration voltage of $12 \mathrm{kV}$. The desired $\mathrm{W}^{17+}$ ions were selected by a $90^{\circ}$ dipole magnet set to a suitable magnetic field. Additional charge-state cleaning of the beam was achieved by a $90^{\circ}$ electrostatic spherical deflector in front of the electron-ion collision region. Behind the deflector the ion beam was collimated by two slits separated from one another by $195 \mathrm{~mm}$ and set to a square opening between $0.8 \times 0.8 \mathrm{~mm}^{2}$ and $2 \times 2 \mathrm{~mm}^{2}$. The ion beam was crossed under $90^{\circ}$ with an intense ribbon shaped electron beam [21] at energies of up to $1000 \mathrm{eV}$. In the electron-ion collision region electron-impact ionization occurred. The ionized product ions were separated from the primary beam by a second $90^{\circ}$ dipole magnet and a subsequent $180^{\circ}$ out-of-plane electrostatic deflector. The product ions were detected by a single particle detector $[22,23]$ while the current of the primary beam was measured by a movable Faraday cup.

Measurements of absolute cross sections were performed employing the animatedbeams technique $[24,25]$ where the electron gun is moved (in $z$-direction) perpendicular to the plane defined by the ion and the electron beam from a position on one side of the ion beam with no overlap via optimum overlap of both beams to the opposite side of the ion beam where there is no overlap again. This procedure allows one to measure the background count rate at no-overlap positions and to determine a $z$-dependent ionization signal on top of that background at positions where there is overlap. While the electron gun as a whole is moved by a motor the count rate $R$ seen by the product-ion detector, the ion current $I_{i o n}$, the electron current $I_{e}$ and the dwell time $t$ at positions $z^{j}$ are recorded and accumulated in channels $j$ of multi-channel analyzer spectra. For this purpose the ion current is converted (with a conversion factor $C_{i o n}$ ) to a train of pulses the frequency of which is proportional to the ion current. Similarly, the electron current is converted with a conversion factor $C_{e}$. Time is represented by the frequency $r_{\text {time }}$ of pulses from a constant-rate pulse generator. The total distance of about $20 \mathrm{~mm}$ 
traveled by the electron gun is divided into individual bins of height $\Delta z \approx 40 \mu \mathrm{m}$ and four multi-channel spectra are generated by accumulating the counts from the ion detector and the pulses from the ion and electron current converters together with the pulser signals in up to 512 channels for each spectrum. The width of each channel $j$ corresponds to $\Delta z$, the channel number $j$ itself corresponds to the position $z=z^{j}$ of the electron gun with respect to the ion beam axis. After the measurement a fifth spectrum is generated by dividing (channel by channel) the signal spectrum by the ion and electron current spectra and multiplying the result by the time spectrum. The new spectrum then contains normalized signal-plus-background counts.

An example for a complete set of spectra taken for an absolute cross section measurement is shown in figure 1. The spectrum in panel 1 contains the numbers of signal+background counts $N_{\text {sig }}^{j}=R \tau^{j}$ for channel numbers $j$ where $\tau^{j}$ is the total dwell time at position $z^{j}$. The observed peak is due to electron-impact single ionization of $\mathrm{W}^{17+}$ when the two beams overlap. The spectrum in panel 2 with $N_{i o n}^{j}=C_{i o n} I_{i o n} \tau^{j}$ represents the accumulated ion charge per channel. The spectrum in panel 3 with $N_{e}^{j}=C_{e} I_{e} \tau^{j}$ represents the accumulated electron charge per channel. The dwell time per channel is recorded in spectrum 4 where the number of counts in channel $j$ is given by $N_{\text {time }}^{j}=r_{\text {time }} \tau^{j}$. The pulse rate was $r_{\text {time }}=10^{6} \mathrm{~s}^{-1}$, the dwell time per channel about $5 \mathrm{~s}$ in total and the number of sweeps across the ion beam accumulated in about 40 minutes was more than 100.

The absolute electron-impact ionization cross section can be calculated $[21,25,26]$ from the position-dependent normalized signal as

$$
\sigma=S \frac{\Delta z}{M \varepsilon} \frac{C_{i o n} C_{e}}{r_{\text {time }}}
$$

where $S$ is the area under the signal peak in the fifth spectrum described above. The kinematic factor $M$ in a $90^{\circ}$ crossed-beams experiment is defined as

$$
M=\sqrt{v_{i o n}^{2}+v_{e}^{2}} /\left(v_{i o n} v_{e} q_{i o n} e^{2}\right)
$$

where $v_{i o n}$ and $v_{e}$ are the ion and electron velocities, respectively, $q_{i o n}$ is the charge state of the ion and $e$ is the elementary charge. The efficiency of the signal detector is quantified by $\varepsilon$. Numerically, $S$ is determined from the spectrum of normalized counts stored in channels $j=1, \ldots, 512$ (panel 5 in figure 1)

$$
N_{\text {norm }}^{j}=\frac{N_{\text {sig }}^{j} N_{\text {time }}^{j}}{N_{\text {ion }}^{j} N_{e}^{j}} .
$$

With the subtraction of the average background per channel $\bar{N}_{b g r}, S$ follows from

$$
S=\sum_{j}\left(N_{n o r m}^{j}-\bar{N}_{b g r}\right) .
$$

The derivation of this concept and the robustness of the technique have been discussed in detail in reference [27]. It should be noted that the determination of the cross section does not require any additional information about the overlap form factors. 


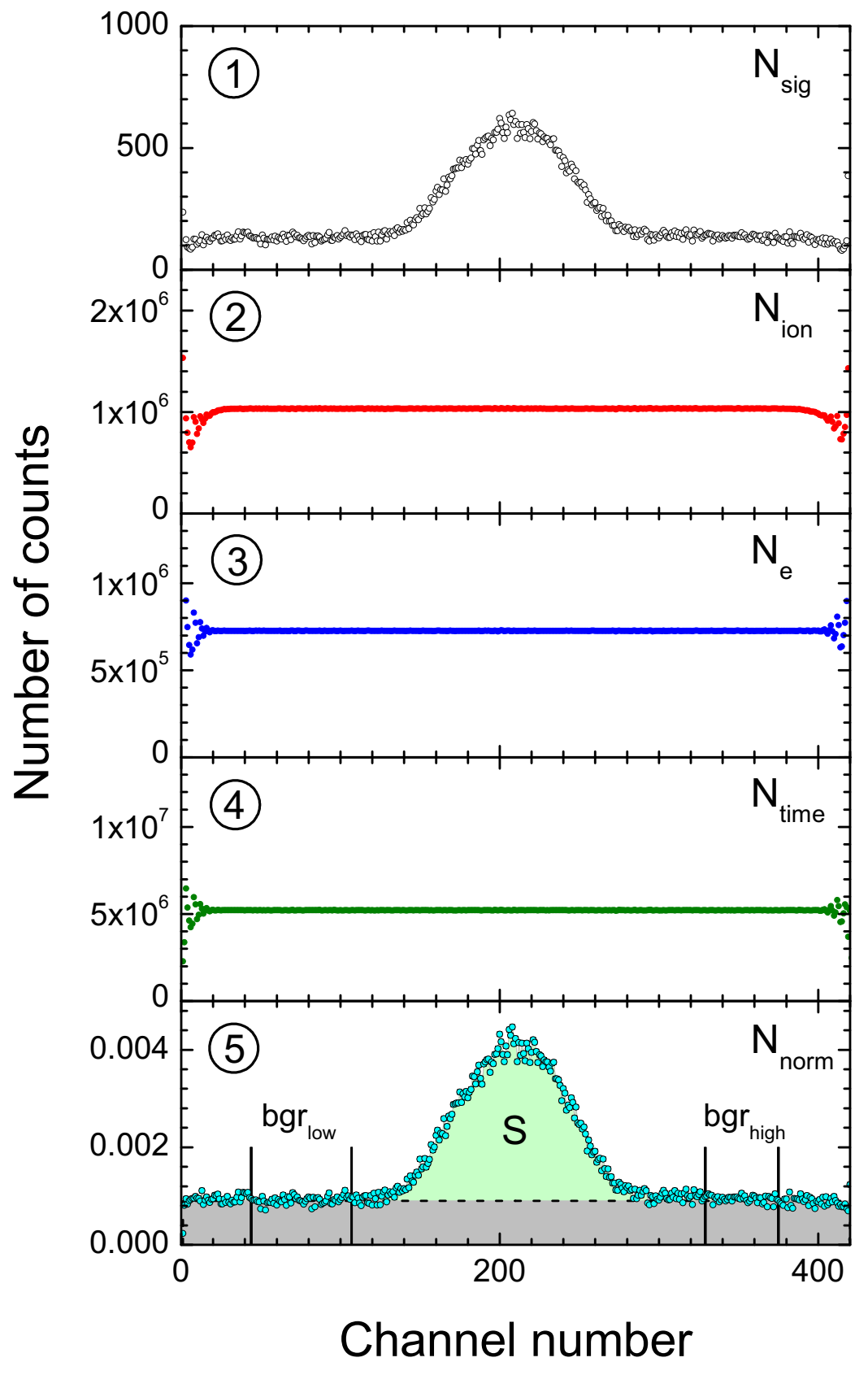

Figure 1. Raw data of an absolute cross section measurement for ionization of $\mathrm{W}^{17+}$ at $449 \mathrm{eV}$ electron-ion collision energy. The spectra contain signal+background counts $N_{\text {sig }}$ (panel 1), a measure of the ion charge $N_{i o n}$ (panel 2) accumulated per channel, a measure of the electron charge $N_{e}$ (panel 3) accumulated per channel, and the dwell time $N_{\text {time }}$ per channel (panel 4). The spectrum in panel 5 is obtained by normalizing spectrum 1 to spectra 2 and 3 and multiplying with spectrum 4 (see text). Average background is determined from the two regions $b g r_{l o w}$ and $b g r_{h i g h}$ distinguished by the vertical markers displayed in panel 5. After subtraction of the average background the signal peak area $S$ remains which is proportional to the cross section to be determined.

High-resolution energy-scan spectra can be obtained in a second mode of operation 
of the present crossed-beams setup where the position of the gun is kept at maximum overlap of the two beams. The electron energy is varied in small steps and the signal+background count rate is recorded together with the ion and electron currents and the data accumulation time per channel. With less than $300 \mu$ s necessary for all the voltages on the electron gun to settle after initialization of an energy step, dwell times on each energy as low as $3 \mathrm{~ms}$ can be chosen at $90 \%$ duty cycle. Sweeps over a pre-defined energy range can span up to $200 \mathrm{eV}$ with up to 4096 steps. Given the energy spread of the electron beam (of less than $0.5 \mathrm{eV}$ at energies below $100 \mathrm{eV}$ ) the smallest meaningful energy steps are of the order of about $0.05 \mathrm{eV}$. The energy sweeps are repeated until the desired statistical uncertainty of the detector signal is reached. Scans over wider energy ranges are measured in pieces of $200 \mathrm{eV}$ width overlapping one another by $100 \mathrm{eV}$. The complete experimental ionization spectrum is obtained by appropriate averaging of the data from adjacent overlapping scan ranges. Finally, the complete scan spectrum is normalized to the absolute cross sections measured separately by using the animated beams method.

\subsection{Experimental parameters specific to the present experiment}

The $\mathrm{W}^{17+}$ ions produced in the ECR ion source were accelerated by a voltage of $12 \mathrm{kV}$. At this voltage it was possible to extract ${ }^{184} \mathrm{~W}^{17+}$ ions with electrical currents between $82 \mathrm{nA}$ and $94 \mathrm{nA}$ from the ion source using oxygen as a support gas. The ${ }^{184} \mathrm{~W}^{17+}$ ion beam was collimated to a size of $2 \times 2 \mathrm{~mm}^{2}$ retaining about $20 \mathrm{nA}$ for energy-scan measurements. The signal-to-noise ratio for the single-ionization scan measurements was 0.25 at an electron energy of $415 \mathrm{eV}$ and 17 at an electron energy of $1000 \mathrm{eV}$. In the case of double ionization the signal-to-noise ratio was 16 at an electron energy of $900 \mathrm{eV}$ and 140 at $1000 \mathrm{eV}$. For absolute measurements the beam was collimated to a size of $0.8 \times 0.8 \mathrm{~mm}^{2}$ while retaining about $4 \mathrm{nA}$ ion current. For single ionization this resulted in a signal-to-noise ratio of 0.47 at $415 \mathrm{eV}$ electron energy up to 33 at $1000 \mathrm{eV}$ electron energy. In the case of double ionization the signal-to-noise ratio changed to 33 at $900 \mathrm{eV}$ and 280 at $1000 \mathrm{eV}$.

The physical separation of the $\mathrm{A}^{(q+1)+}$ product ion beam from the $\mathrm{A}^{q+}$ primary ion beam becomes increasingly more difficult with increasing $q$. The Faraday cup which collects the primary ion beam has to be placed closer to the product ion beam when the charge state $q$ increases. In the present setup, the properly positioned Faraday cup may intercept parts of the product ion beam for $q \geq 18$. For single ionization of an ion in charge state $q=17$ the requirements of simultaneous complete collection of the primary ions in the Faraday cup and complete detection of the ionized ions can still be guaranteed but is not easily achieved. By carefully positioning the Faraday cup it could be shown that at most $1.5 \%$ of the parent ion current might have missed the Faraday cup when all the ionization signal was collected on the product-ion detector.

The possible systematic error of the absolute measurements is estimated from the quadrature sum of the systematic uncertainties which enter the cross section calculation 
in equation 1 . These are the kinematic factor with $1 \%$ uncertainty [21], the detector efficiency with $3 \%$ [22], the $\Delta z$ displacement of the electron gun with $1 \%$, the ion current measurement with $5 \%$. For single ionization cross sections of $\mathrm{W}^{17+}$ the additional $1.5 \%$ uncertainty of the parent ion current is also considered. In contrast to the previous estimate of possible errors [21] the systematic uncertainty of the electron current was reduced from $5 \%$ to $2 \%$, however, an additional energy dependent electron current correction function was introduced [17] which has an energy-dependent uncertainty of about $15 \%$ at $20 \mathrm{eV}$ and less than $1 \%$ at energies greater than $120 \mathrm{eV}$. The correction accounts for the energy-dependent shape of the electron beam and resultant loss of current to the electron gun electrodes before the electron beam enters the interaction region.

The error budget comprises an energy-independent systematic error of $6.5 \%$ for single ionization of $\mathrm{W}^{17+}$ and of $6.3 \%$ for double ionization. In addition, the total uncertainty of the measured cross sections takes into account a possible error for a deadtime correction of the single particle detector, the statistical error of the signal count rate and the uncertainty of the energy-dependent electron current correction function. In the energy range from $450-1000 \mathrm{eV}$ the total error is about $8.3 \%$ for both, the single- and the double-ionization measurements. The scan spectra are normalized to the absolute measurements. Therefore, their uncertainty is mainly determined by the total error bar of the absolute cross sections.

\section{Results}

\subsection{Single Ionization}

Absolute cross sections for the single ionization of $\mathrm{W}^{17+}$ ions have been obtained at electron-ion collision energies between $375 \mathrm{eV}$ and $1000 \mathrm{eV}$. Scan measurements with a step width of $0.2 \mathrm{eV}$ where performed at energies between $300 \mathrm{eV}$ and $1000 \mathrm{eV}$. The absolute data and the normalized energy-scan spectrum are shown in figure 2.

Two absolute measurements of the cross section have been carried out at energies (350 eV and $375 \mathrm{eV}$ ) below the ground-state ionization threshold. No statistically significant net signal was found at these energies. Observation of signal below the ground state ionization threshold would indicate the presence of ions in long-lived excited states in the parent $\mathrm{W}^{17+}$ ion beam. The measured ionization cross section is zero up to about $402 \mathrm{eV}$. From there on it begins to rise with an increasing slope as shown by the energyscan measurement displayed in figure $2 \mathrm{~b}$ ). The cross section gets steeper from about $402 \mathrm{eV}$ to $418 \mathrm{eV}$ where a linear energy dependence up to about $460 \mathrm{eV}$ sets in. Above $470 \mathrm{eV}$ the slope of the measured cross section decreases until it levels off. The maximum single-ionization cross section is $3.0 \cdot 10^{-18} \mathrm{~cm}^{2}$ at about $900 \mathrm{eV}$. No clear structures such as resonances or excitation-autoionization edges can be seen in the cross section.

According to Kramida and Shirai [28] the ground state of $\mathrm{W}^{17+}$ has a $[\mathrm{Kr}] 4 \mathrm{~d}^{10} 4 \mathrm{f}^{11}$ configuration with an ionization energy of $420.7 \pm 1.4 \mathrm{eV}$. Calculations with the Los 

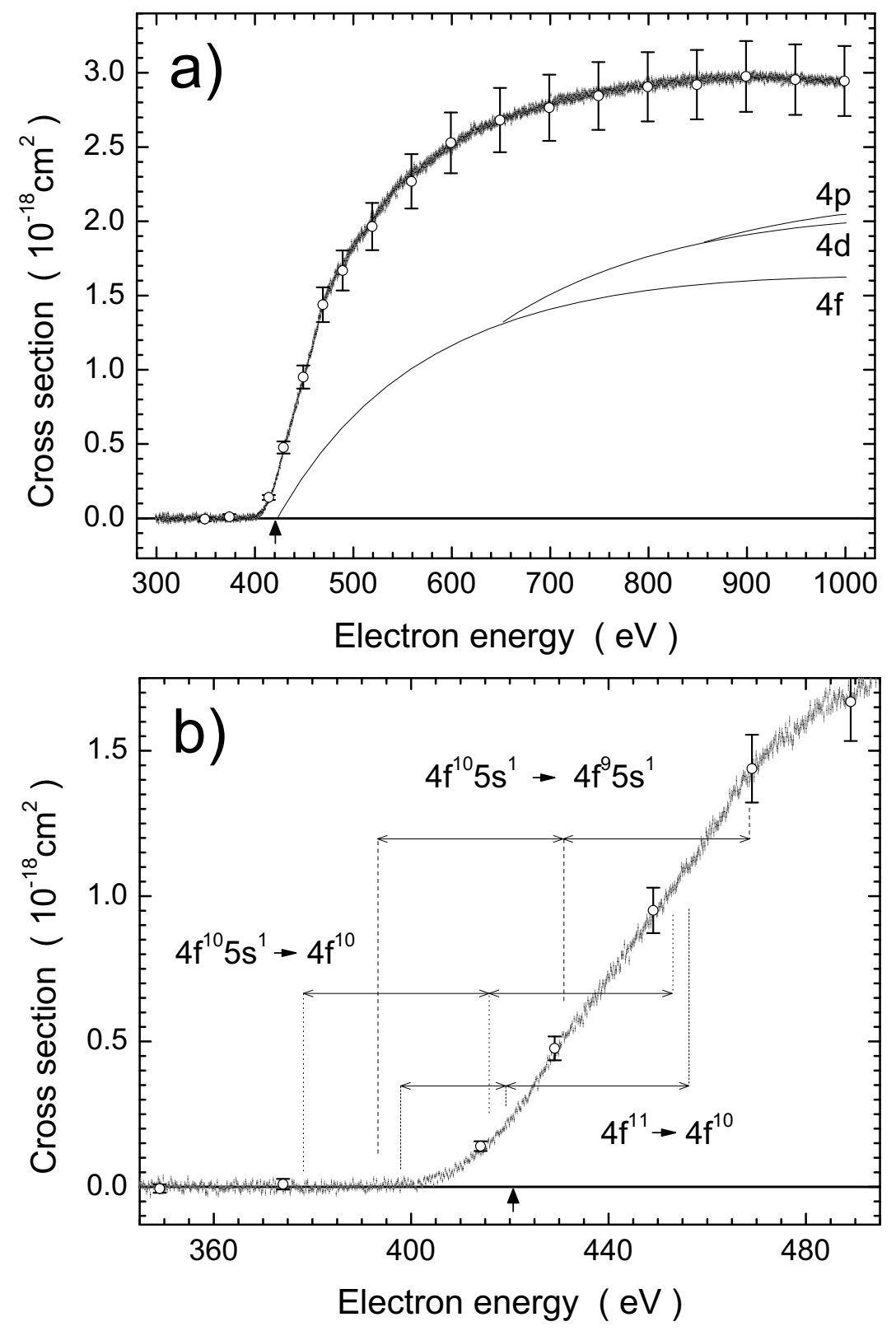

Figure 2. a) Cross section for single ionization of $\mathrm{W}^{17+}$. The solid lines in a) represent the summed calculated cross sections for direct single ionization of the $4 f, 4 d$ and $4 p$ subshells. The open circles show results of absolute cross section measurements, the small gray vertical bars represent the statistical uncertainties of the scan measurements. The vertical arrow in a) and in b) marks the ionization threshold calculated by Kramida and Shirai [28]. The threshold region of single ionization of $\mathrm{W}^{17+}$ is shown in b). Horizontal arrows represent the energy regions in which the indicated processes, such as $4 f^{11} \rightarrow 4 f^{10}$, can occur. In each case the left arrow shows the region in which a metastable fine structure state from the initial configuration can be ionized to the ground state of the $\mathrm{W}^{18+}$ ion. The right arrow marks the energy range for ionization resulting in one of the fine structure states within the specified product configuration of $\mathrm{W}^{18+}$. 
Alamos National Laboratory (LANL) atomic physics code package [29] in the finestructure mode yield an ionization energy of $419.2 \mathrm{eV}$. These numbers are consistent with the start of the linear increase of the cross section above $418 \mathrm{eV}$. For an assessment of cross section contributions originating from direct knock-off ionization and indirect processes involving excitation and subsequent autoionization, calculations were performed, again employing the LANL code package. Cross sections for direct single ionization of the $4 p, 4 d$ and $4 f$ subshells were calculated using the configurationaverage distorted-wave approximation. The dominant contribution to the total direct ionization is associated with the $4 f$ shell. The calculated cross section is shown in figure 2 a) with the contributions originating from the $4 d$ and $4 p$ subshells added on top. The measured total single ionization cross section rises more steeply than the calculated direct single-ionization cross section. It reaches a local maximum at about $900 \mathrm{eV}$ where the calculated direct ionization cross section is still increasing and at $1000 \mathrm{eV}$ the experimental cross section is still about $45 \%$ above the calculated cross section. This may partly be due to an underestimation of direct ionization by theory but more likely the difference has to be attributed to the presence of indirect ionization processes which are not included in the calculation.

The rising slope of the cross section between $402 \mathrm{eV}$ and $418 \mathrm{eV}$ shown in figure $2 \mathrm{~b}$ ) has a real physical origin and cannot be explained as an artifact due to experimental energy spread which is only $2.5 \pm 0.5 \mathrm{eV}[12,26]$ in the present range of electron-ion collision energies. It is probably caused by the unresolved thresholds of the 41 fine structure levels of the $4 f^{11}$ ground state configuration. Ions in these states must be expected to be present in the parent ion beam. According to the LANL code package the states of the ground state configuration cover an energy range of $21.3 \mathrm{eV}$, i.e., their ionization thresholds are expected between $397.9 \mathrm{eV}$ and $419.2 \mathrm{eV}$.

The linear increase of the cross section above the ground state ionization energy might be influenced by additional ionization channels opening up as the electron energy increases. The LANL code package predicts a minimum ground state ionization threshold of $419.2 \mathrm{eV}$ for the ionization process forming $\mathrm{W}^{18+}\left(4 f^{10}\right)$. The $4 f^{10}$ ground state configuration of $\mathrm{W}^{18+}$ features 107 fine structure levels within an energy range of $37.1 \mathrm{eV}$ between 419.2 and $456.3 \mathrm{eV}$. In this range, ionization into an increasing number of exited $4 f^{10}$ fine structure states becomes possible as the electron energy increases. Figure $2 \mathrm{~b}$ ) shows these energy ranges labeled as $4 f^{11} \rightarrow 4 f^{10}$ for the ionization of the $4 f^{11}$ ground state configuration of $\mathrm{W}^{17+}$ to the $4 f^{10}$ ground state configuration of $\mathrm{W}^{18+}$.

Besides ions in states belonging to the $\mathrm{W}^{17+}\left(4 f^{11}\right)$ configuration metastable ions in the excited $4 f^{10} 5 s^{1}$ configuration might have also been present in the parent ion beam. The $4 f^{10} 5 s^{1}$ configuration comprises 208 fine structure levels spread out over an energy range of $37.6 \mathrm{eV}$. Removal of the $5 \mathrm{~s}$ electron from one of these states is possible between 378.2 and $415.8 \mathrm{eV}$. If one considers in addition the fine structure splitting of the resulting $4 f^{10}$ configuration then the possible ionization thresholds can reach up to $452.9 \mathrm{eV}$ as indicated in figure $2 \mathrm{~b}$ ) by the energy ranges labeled as $4 f^{10} 5 s^{1} \rightarrow 4 f^{10}$. However, no evident signature of these processes can be seen in the measurement. In 
particular, the detectable ionization onset in the experiment is about $20 \mathrm{eV}$ above the calculated lowest ionization threshold of the first excited configuration of the parent $\mathrm{W}^{17+}$ ion. One can expect, that removal of one of the ten $4 f$ electrons of the $4 f^{10} 5 s^{1}$ configuration is much more probable than $5 s$-ionization. Going from any state of the $4 f^{10} 5 s^{1}$ configuration of $\mathrm{W}^{17+}$ to the ground state of $\mathrm{W}^{18+}$ requires electron energies in the range between $393.2 \mathrm{eV}$ and $430.8 \mathrm{eV}$. Additional channels for the ionization into exited states within the $4 f^{9} 5 s^{1}$ configuration of $\mathrm{W}^{18+}$ cover energies up to $468.6 \mathrm{eV}$ as indicated in figure $2 \mathrm{~b}$ ) by the energy ranges labeled as $4 f^{10} 5 s^{1} \rightarrow 4 f^{9} 5 s^{1}$. Above that energy the slope of the cross section function diminishes. This might be interpreted as an indication for an admixture of metastable $4 f^{10} 5 s^{1}$ ions in the parent $\mathrm{W}^{17+}$ ion beam.

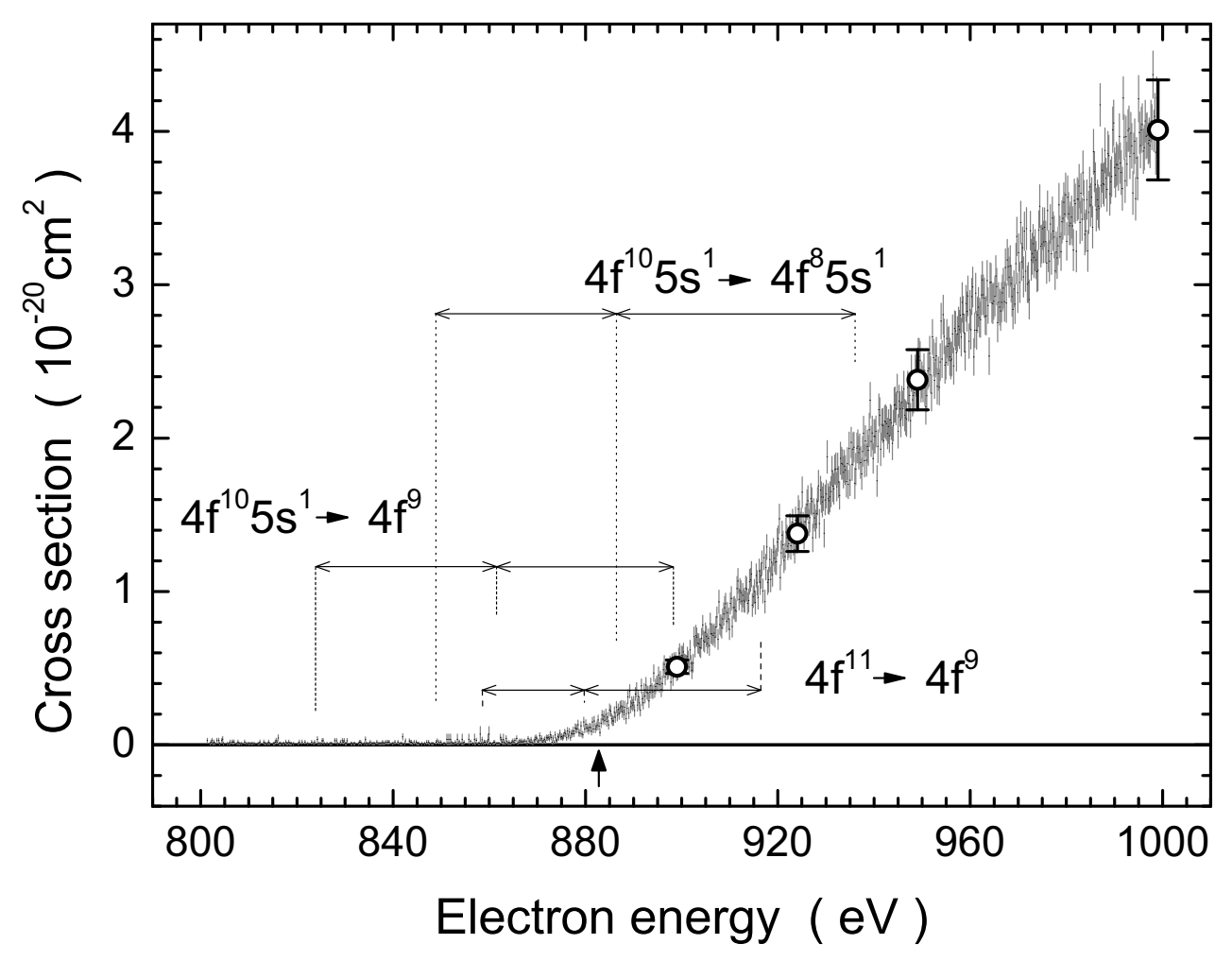

Figure 3. Experimental cross sections for double ionization of $\mathrm{W}^{17+}$. The notation is the same as in figure 2 .

\subsection{Double Ionization}

For the double ionization of $\mathrm{W}^{17+}$ ions absolute measurements were performed at electron-ion collision energies between 900 and $1000 \mathrm{eV}$. In addition, an energy-scan measurement was carried out in the energy range 800 to $1000 \mathrm{eV}$. Figure 3 shows the absolute cross sections and the normalized scan spectrum. In the relatively narrow energy range that could be accessed around the double ionization threshold no structure in the energy dependence of the cross section can be observed. 
The ionization threshold of $882.8 \pm 2.0 \mathrm{eV}$ indicated by the vertical arrow in figure 3 has been determined on the basis of the single-ionization potentials calculated by Kramida and Shirai [28] for all charge states of tungsten. The calculations carried out using the LANL code package [29] result in a threshold of $879.8 \mathrm{eV}$. The experimental cross section has its onset at about $870 \mathrm{eV}$ from where the slope is rapidly increasing. Above $900 \mathrm{eV}$ the cross section increases with a constant slope. Similar to figure $2 \mathrm{~b}$ ), which covers the threshold region of single ionization, figure 3 shows the energy ranges for the ionization of the ground state and first-excited-state configurations to all states in the indicated $\mathrm{W}^{19+}$ product configurations. As in the case of single ionization of $\mathrm{W}^{17+}$ the smooth onset of the double ionization cross section below the ground state ionization threshold probably has its origin in the 41 fine structure levels of the $4 f^{11}$ ground state configuration or in the 208 levels of the $4 f^{10} 5 s^{1}$ configuration of the $\mathrm{W}^{17+}$ parent ions. Additional ionization channels of the 189 states of the $4 f^{9}$ or the 576 states of the $4 f^{8} 5 s^{1}$ configuration may also result in the observed smooth progression of the cross section towards higher electron-ion collision energies.

\section{Summary}

In the present work the feasibility of producing up to $94 \mathrm{nA}$ of ${ }^{184} \mathrm{~W}^{17+}$ ions from tungsten hexacarbonyl vapor in an all-permanent-magnet ECR ion source is demonstrated. This ion current is well sufficient for performing single- and doubleionization cross section measurements using our crossed-beams setup. For single ionization the comparison of calculated and measured cross sections indicates a significant role of indirect processes. Calculated ionization thresholds were found to be in quite good agreement with theoretical expectations. Numerous individual states within the ground-state and first excited-state configurations produce cross-section onsets spanning very wide energy ranges of the order of $50 \mathrm{eV}$. Our results contribute to the ongoing effort to build a reliable atomic-data base satisfying the needs of fusion science.

\section{Acknowledgments}

The authors gratefully acknowledge support by Deutsche Forschungsgemeinschaft under project number $\mathrm{Mu}$ 1068/20.

\section{References}

[1] Bolt H, Barabash V, Krauss W, Linke J, Neu R, Suzuki S, Yoshida N and the ASDEX Upgrade Team 2004 Journal of Nuclear Materials 329-333 66-73

[2] Dux R, Janzer A, Pütterich T and the ASDEX Upgrade Team 2011 Nucl. Fusion 51053002

[3] Radtke R, Biedermann C, Mandelbaum P and Schwob J L 2007 Journal of Physics: Conference Series $\mathbf{5 8}$ 113-116

[4] Pütterich T, Neu R, Dux R, Whiteford A D, O'Mullane M G, Summers H P and the ASDEX Upgrade Team 2010 Nucl. Fusion 50025012 
[5] Müller A 2008 Adv. At. Mol. Phys. 55 293-417

[6] Schippers S, Bernhardt D, Müller A, Krantz C, Grieser M, Repnow R, Wolf A, Lestinsky M, Hahn M, Novotný O and Savin D W 2011 Phys. Rev. A 83012711

[7] Müller A, Schippers S, Kilcoyne A L D and Esteves D 2011 Phys. Scr. In the press

[8] Montague R G and Harrison M F A 1984 J. Phys. B 172707 - 2711

[9] Stenke M, Aichele K, Harthiramani D, Hofmann G, Steidl M, Völpel R and Salzborn E $1995 J$. Phys. B 28 2711-2721

[10] Stenke M, Aichele K, Harthiramani D, Hofmann G, Steidl M, Völpel R, Shevelko V P, Tawara H and Salzborn E 1995 J. Phys. B 284853 - 4859

[11] Loch S D, Ludlow J A, Pindzola M S, Whiteford A D and Griffin D C 2005 Phys. Rev. A 72 052716

[12] Teng H, Knopp H, Ricz S, Schippers S, Berrington K A and Müller A 2000 Phys. Rev. A 61060704

[13] Jacobi J, Knopp H, Schippers S, Müller A, Loch S D, Witthoeft M, Pindzola M S and Ballance C P 2004 Phys. Rev. A $\mathbf{7 0} 042717$

[14] Becker C, Knopp H, Jacobi J, Teng H, Schippers S and Müller A 2004 J. Phys. B 37 1503-1518

[15] van Zoest T, Knopp H, Jacobi J, Schippers S, Phaneuf R A and Müller A 2004 J. Phys. B 37 $4387-4395$

[16] Jacobi J, Knopp H, Schippers S, Shi W and Müller A 2005 J. Phys. B 38 2015-2028

[17] Borovik Jr A, Müller A, Schippers S, Bray I and Fursa D V 2009 J. Phys. B 42025203

[18] Renwick A C, Bray I, Fursa D V, Jacobi J, Knopp H, Schippers S and Müller A 2009 J. Phys. B 42175203

[19] Brötz F, Trassl R, McCullough R W, Arnold W and Salzborn E 2001 Phys. Scr. T92 278-280

[20] Lai K K and Lamb H H 2000 Thin Solid Films $370114-121$

[21] Müller A, Huber K, Tinschert K, Becker R and Salzborn E 1985 J. Phys. B 18 2993-3009

[22] Rinn K, Müller A, Eichenauer H and Salzborn E 1982 Rev. Sci. Instrum. 53 829-837

[23] Fricke J, Müller A and Salzborn E 1980 Nucl. Instrum. Methods 175 379-384

[24] Defrance P, Brouillard F, Claeys W and Wassenhove G V 1981 J. Phys. B 14 103-110

[25] Müller A, Tinschert K, Achenbach C, Becker R and Salzborn E 1985 Nucl. Instrum. Methods B $10204-206$

[26] Müller A, Teng H, Hofmann G, Phaneuf R A and Salzborn E 2000 Phys. Rev. A 62062720

[27] Müller A 1991 Ion formation processes: Ionization in ion-electron collisions Physics of Ion-Impact Phenomena (Springer Series in Chemical Physics vol 54) ed Mathur D (Berlin, Heidelberg, New York: Springer) pp 13-90

[28] Kramida A and Shirai T 2009 At. Data Nucl. Data Tables 95 305-474

[29] Atomic and Optical Theory Group, Los Alamos National Laboratory (LANL), "Atomic physics codes package", (available at http://aphysics2.lanl.gov/cgi-bin/ION/runlanl08d.pl) 